\title{
National Trends in Visit Rates and Antibiotic Prescribing for Children With Acute Sinusitis
}

\begin{abstract}
WHAT'S KNOWN ON THIS SUBJECT: Streptococcus pneumoniae
is a common cause of acute sinusitis and otitis media, and American Academy of Pediatrics guidelines recommend amoxicillin as the first-line antibiotic therapy. Amoxicillin use for otitis media increased after guideline publication, and visits decreased after vaccine introduction.

WHAT THIS STUDY ADDS: Office and emergency department visits for acute sinusitis remained stable after vaccine introduction, whereas amoxicillin use increased substantially, in accordance with the guidelines. Despite the increase in amoxicillin use, prescriptions for broad-spectrum agents, especially macrolides, remain common and unnecessary.
\end{abstract}

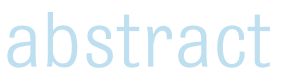

OBJECTIVE: The heptavalent pneumococcal conjugate vaccine contributed to a substantial decrease in the number of ambulatory visits attributable to acute otitis media (AOM) and amoxicillin use for AOM increased after publication of American Academy of Pediatrics guidelines regarding AOM. Our objective was to determine whether similar trends occurred for children with acute sinusitis.

METHODS: We analyzed data from the National Ambulatory Medical Care Survey and National Hospital Ambulatory Medical Care Survey (1998-2007), which are nationally representative surveys of office and emergency department visits. For children younger than 18 years with diagnosed acute sinusitis $(N=538)$, we examined time trends in visit rates and antibiotic prescribing. Multivariate logistic regression analyses were used to identify factors associated with narrow-spectrum antibiotic prescribing.

RESULTS: Between 1998 and 2007, the annual visit rate for acute sinusitis remained stable, ranging from 11 to 14 visits per 1000 children $(P=.67)$. No change occurred in the proportion of visits with receipt of an antibiotic (82\%; $P=.71$ ); however, the proportion with receipt of amoxicillin increased from $19 \%$ to $58 \%$ during the study period ( $P<$ .01). Prescriptions for broader-spectrum agents, especially macrolides (18\% overall), remained common.

CONCLUSIONS: Unlike the visit rate for AOM, the visit rate for acute sinusitis among children did not decrease after introduction of the pneumococcal conjugate vaccine. Although prescriptions for amoxicillin increased in accordance with the guidelines, reducing unnecessary prescriptions for macrolides remains an important target for campaigns promoting judicious antibiotic use. Pediatrics 2011;127:28-34
AUTHORS: Daniel J. Shapiro, BA, a,b Ralph Gonzales, MD, MSPH, ${ }^{c}$ Michael D. Cabana, MD, MPH, ${ }^{a, b}$ and Adam L. Hersh, MD, PhD a,b,d

Divisions of ${ }^{a}$ General Pediatrics and ${ }^{d}$ Pediatric Infectious Diseases, Department of Pediatrics, ${ }^{b}$ Philip R. Lee Institute for Health Policy Studies, and ${ }^{c}$ Division of General Internal Medicine, Department of Internal Medicine, School of Medicine, University of California, San Francisco, California

KEY WORDS

sinusitis, antibiotic use, physician practice patterns

\section{ABBREVIATIONS}

AAP-American Academy of Pediatrics

AOM - acute otitis media

PCV7-heptavalent pneumococcal conjugate vaccine NCHS - National Center for Health Statistics NAMCS - National Ambulatory Medical Care Survey NHAMCS — National Hospital Ambulatory Medical Care Survey $\mathrm{Cl}$-confidence interval OR—odds ratio

www.pediatrics.org/cgi/doi/10.1542/peds.2010-1340

doi:10.1542/peds.2010-1340

Accepted for publication 0ct 1, 2010

Address correspondence to Adam L. Hersh, MD, PhD, University of Utah, Pediatric Infectious Diseases, 295 Chipeta Way, Salt Lake City, UT 84108. E-mail: adam.hersh@hsc.utah.edu

PEDIATRICS (ISSN Numbers: Print, 0031-4005; Online, 1098-4275) Copyright $\odot 2011$ by the American Academy of Pediatrics FINANGIAL DISCLOSURE: The authors have indicated they have no financial relationships relevant to this article to disclose. Funded by the National Institutes of Health $(\mathrm{NIH})$. 
Acute sinusitis is a common condition in ambulatory care, accounting for $>3$ million visits to ambulatory care settings per year among patients of all ages. ${ }^{1}$ Because physicians routinely prescribe antibiotics for patients with acute sinusitis, it is an important source of antibiotic consumption, some of which may be unnecessary. ${ }^{1-3}$

Streptococcus pneumoniae is a common bacterial cause of both acute sinusitis and acute otitis media (AOM) ${ }^{4}$ Accordingly, the American Academy of Pediatrics (AAP) clinical practice guidelines for acute sinusitis (2001) and AOM (2004) recommend amoxicillin as the first-line antibiotic for both infections. Amoxicillin-clavulanate and cephalosporins, which are broaderspectrum agents, are recommended only for selected patients (eg, in severe cases or cases with previous treatment failure), whereas macrolides are not routinely recommended for acute sinusitis. 5,6

During the past decade, the incidence and antibiotic treatment of AOM have changed considerably. Introduction of the heptavalent pneumococcal conjugate vaccine (PCV7) in 2000 has contributed to a substantial decrease in the rate of ambulatory visits attributable to A0M.,7,8 In addition, use of guideline-recommended amoxicillin increased after publication of AAP practice guidelines for AOM in 2004, ${ }^{9}$ reversing an earlier trend of increased use of broad-spectrum antibiotics for AOM. ${ }^{10}$

Because of the similarities between acute sinusitis and AOM in terms of bacterial causes and treatment recommendations, we hypothesized that, after introduction of PCV7 in 2000 and publication of clinical practice guidelines for acute sinusitis in 2001, trends in visit rates and antibiotic prescribing for acute sinusitis would be similar to those observed for AOM.

\section{METHODS}

\section{Data Source and Design}

We analyzed data from the National Ambulatory Medical Care Survey (NAMCS) and the National Hospital Ambulatory Medical Care Survey (NHAMCS) for a 10year period between 1998 and 2007, to estimate time trends in visit rates and antibiotic prescribing for acute sinusitis in children. This period encompassed the introduction of PCV7 and publication of AAP guidelines regarding acute sinusitis. These cross-sectional surveys are conducted annually by the National Center for Health Statistics (NCHS) and provide nationally representative data on patient visits to offices, hospital outpatient departments, and emergency departments. From the surveys, the NCHS compiles physician-level data, including specialty, practice location, and setting, as well as patient-level information such as demographic features, diagnoses (on the basis of International Classification of Diseases, Ninth Revision, Clinical Modification, codes), and medications prescribed. ${ }^{11}$

Data from the NAMCS represent patient visits to physicians' offices. This survey uses a 3-stage design consisting of 112 geographic primary sampling units, physician practices within primary sampling units, and patient visits within physician practices. Data from the NHAMCS represent patient visits to hospital outpatient departments and emergency departments. The NHAMCS uses a 4-stage design consisting of geographic primary sampling units, hospitals within primary sampling units, clinics and emergency service areas within hospitals, and patient visits within clinics or emergency service areas. The NCHS provides patient visit weights for the purpose of generating national estimates of visits and associated characteristics. ${ }^{12}$

\section{Visits Attributable to Acute}

\section{Sinusitis}

In our analysis of visits attributable to acute sinusitis, our primary outcome measure was the estimated annual number of visits per 1000 children. We estimated the number of visits for patients $<18$ years of age, as well as for subpopulations consisting of children 0 to 5 years, 6 to 11 years, and 12 to 18 years of age. A diagnosis of acute sinusitis was designated for visits in which any of 3 diagnosis fields contained the International Classification of Diseases, Ninth Revision, Clinical Modification code for acute sinusitis (code 461.x). Concomitant diagnoses that could potentially warrant antibiotics (eg, pharyngitis, otitis media, pneumonia, skin and soft-tissue infections, and urinary tract infections) accounted for small proportions of visits. Exclusion of those visits did not significantly change the overall estimates of office visit rates; therefore, we included them to maximize sample size.

\section{Antibiotic Prescribing}

In our analysis of antibiotic selection, to increase our certainty that the antibiotic prescription was intended to treat acute sinusitis, we applied morestringent criteria and restricted our sample to visits for which acute sinusitis was the primary diagnosis (ie, appearing in the first diagnosis field of the NAMCS or NHAMCS data entry form). In this analysis, our primary outcome measures were (1) the proportion of visits attributable to acute sinusitis in which any antibiotic was prescribed (antibiotic visits) and (2) the proportion of antibiotic visits in which amoxicillin (the recommended first-line antibiotic, according to AAP guidelines) was prescribed. We identified antibiotics on the basis of the following medication names and antibiotic classes (nomenclature adopted from the National Drug Code Directory and the 
Multum Classification ${ }^{13}$ ): first-, second-, and third-generation cephalosporins, macrolides, amoxicillin-clavulanate, amoxicillin, quinolones, and sulfonamides. One patient diagnosed as having acute sinusitis received penicillin, which was included in the amoxicillin category. A secondary outcome was the proportion of visits attributable to acute sinusitis in which broader-spectrum antibiotics were prescribed; these broader-spectrum agents included amoxicillin-clavulanate, secondand third-generation cephalosporins, and macrolides.

Because the unit of observation in both surveys is the visit, we combined estimates of visit rates with US Census denominators to generate populationadjusted estimates of visit rates. In these analyses, census denominators reflect the US resident population as of July 1 in the relevant year. For years before 2000, intercensal estimates were used. ${ }^{14}$

\section{Statistical Analyses}

All statistical analyses were performed by using Stata 11 (Stata Corp, College Station, TX) and accounted for the components of the complex survey design, including patient visit weights, strata, and primary sampling unit design variables. For trends in visit rates, antibiotic visits, and amoxicillin prescribing, we grouped the survey data and census denominators into five 2-year intervals (1998-1999, 2000-2001, 2002-2003, 2004-2005, and 2006-2007), as recommended by the NCHS. For estimates of the use of other agents (amoxicillin-clavulanate, cephalosporins, and macrolides), we examined prescribing during the entire study period and in the years after AAP guideline publication (2002-2007). Trends in visit rates were assessed by using linear tests for trend. Trends in antibiotic selection were assessed by using logistic regression analyses. Sample-size limi- tations precluded analyses of time trends for age-based subpopulations.

We also performed a multivariate logistic regression analysis to determine which patient- and physicianlevel factors were associated with amoxicillin prescribing, as recommended by current clinical guidelines. ${ }^{5}$ In this analysis, we restricted our sample to antibiotic visits attributable to acute sinusitis. Patient- and physicianlevel factors considered were time period (2-year intervals between 19981999 and 2006-2007), race (white or nonwhite), gender, region (Northeast, Midwest, South, or West), insurance type (private, public, or self-pay/ other), setting type (office, outpatient clinic, or emergency department), age (0-5, 6-11, or 12-17 years), and physician specialty (pediatrics, family practice, or emergency medicine/other). In the logistic regression model, we included as independent variables only the patient- and physician-level factors that were at least nominally associated $(P<.2)$ with amoxicillin prescribing. Because there was a high degree of colinearity between patient age and physician specialty (visits made by younger children were more likely to be to pediatricians, whereas visits made by older children were more likely to be to family practice physicians), we examined models in which age was included and specialty excluded and vice versa.

\section{RESULTS}

\section{Visits Attributable to Acute Sinusitis}

During the 10-year period between 1998 and 2007, there were an estimated 8950000 visits (95\% confidence interval [Cl]: $6820000-11090000$ visits) among children with acute sinusitis. This reflects an average of 895000 visits (95\% Cl: $682000-1110000$ visits) per year or $0.4 \%$ (95\% Cl: $0.3 \%-$ $0.5 \%)$ of all ambulatory visits among children during this period. These esti-
TABLE 1 Characteristics of Acute Sinusitis Visits Among Children Younger Than 18 Years in 1998-2007 ( $N=538)$

\begin{tabular}{|c|c|}
\hline Characteristic & $\begin{array}{c}\text { Proportion } \\
\text { of Acute } \\
\text { Sinusitis } \\
\text { Visits, } \% \\
\end{array}$ \\
\hline \multicolumn{2}{|l|}{ Age } \\
\hline $0-5 y$ & 34 \\
\hline $6-11$ y & 24 \\
\hline $12-17$ y & 42 \\
\hline \multicolumn{2}{|l|}{ Gender } \\
\hline Female & 47 \\
\hline Male & 53 \\
\hline \multicolumn{2}{|l|}{ Race } \\
\hline White & 83 \\
\hline Nonwhite & 17 \\
\hline \multicolumn{2}{|l|}{ Physician specialty } \\
\hline Pediatrics & 45 \\
\hline Family practice & 36 \\
\hline Emergency medicine/other & 20 \\
\hline \multicolumn{2}{|l|}{ Insurance type } \\
\hline Private & 75 \\
\hline Medicare or Medicaid & 21 \\
\hline Self-pay/other & 4 \\
\hline \multicolumn{2}{|l|}{ Region } \\
\hline Northeast & 8 \\
\hline Midwest & 25 \\
\hline South & 50 \\
\hline West & 16 \\
\hline \multicolumn{2}{|l|}{ Setting type } \\
\hline Physician's office & 83 \\
\hline Hospital outpatient department & 10 \\
\hline Emergency medicine department & 7 \\
\hline
\end{tabular}

mates were derived from 538 sampled visits in the NAMCS and NHAMCS data sets. Characteristics of visits attributable to acute sinusitis are shown in Table 1.

The annual visit rate for acute sinusitis during this period was stable, ranging from 11 visits ( $95 \% \mathrm{Cl}: 6-15$ visits) to 14 visits (95\% Cl: 9-19 visits) per 1000 population $<18$ years of age (Fig 1 ). There was no time trend in this population-adjusted visit rate $(P=$ $.67)$. The visit rates averaged 13 visits (95\% Cl: 10-16 visits) per 1000 children 0 to 5 years of age, 9 visits (95\% Cl: 5-12 visits) per 1000 children 6 to 11 years of age, and 15 visits $(95 \% \mathrm{Cl}$ : $10-20$ visits) per 1000 children 12 to 17 years of age. 


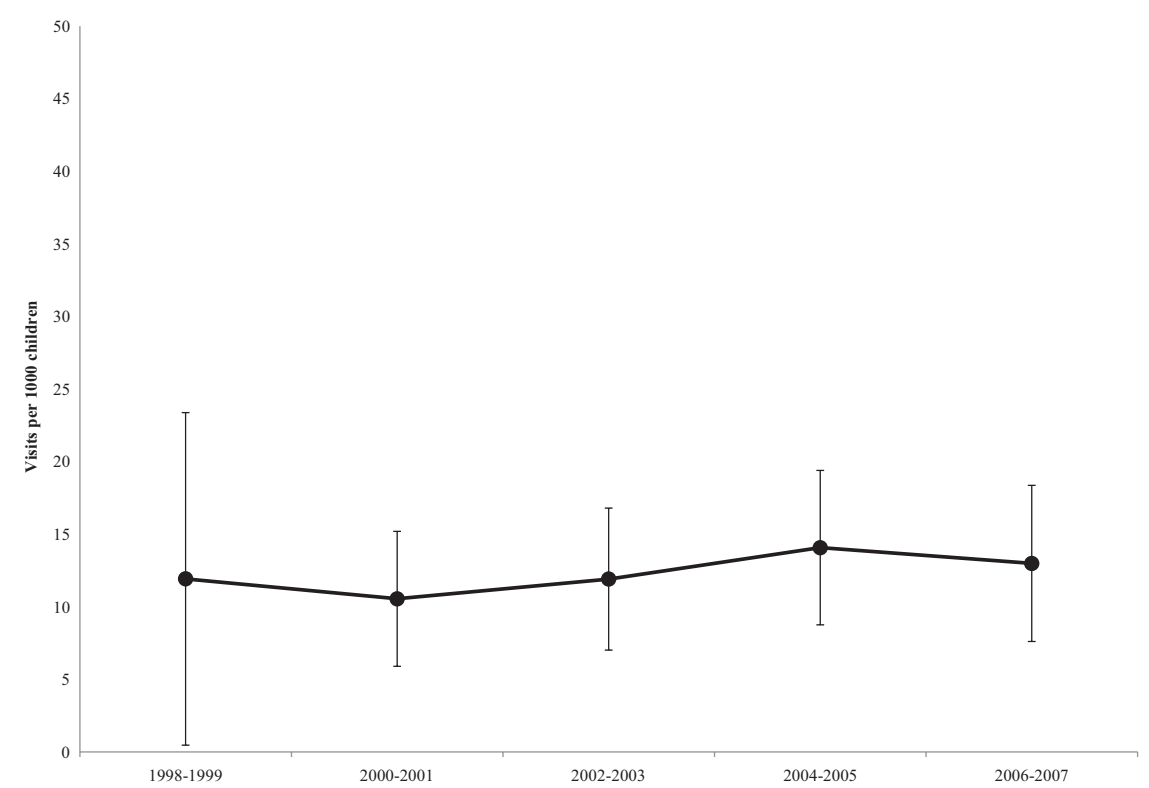

FIGURE 1

Population-adjusted visit rates (estimates and $95 \%$ Cls) for acute sinusitis among children in 19982007. $P=.67$ for trend.

\section{Antibiotic Prescribing}

Antibiotic use for treatment of sinusitis was analyzed in the subset of the sampled visits $(N=389)$ for which the primary diagnosis was acute sinusitis. Antibiotics from any class were prescribed in $82 \%$ (95\% $\mathrm{Cl}: 74 \%-88 \%)$ of visits attributable to acute sinusitis between 1998 and 2007. There was no change in the overall rate at which antibiotics were prescribed $(P=.71)$, which ranged from $76 \%$ (95\% Cl: $54 \%-$ 90\%) to $90 \%$ (95\% Cl: $73 \%-96 \%)$ of acute sinusitis-related visits during this period (Fig 2); however, we observed changes in the use of amoxicillin over time. The proportion of antibiotic visits in which amoxicillin was prescribed increased from 19\% (95\% $\mathrm{Cl}: 10 \%-35 \%)$ to $58 \% \quad(95 \% \quad \mathrm{Cl}$ : $40 \%-74 \%)$ during the study period $(P<.01)$ (Fig 3). Among the antibiotics prescribed as alternatives to amoxicillin, the most commonly prescribed were second- and third-generation cephalosporins $(17 \%$ of antibiotic visits), amoxicillin-clavulanate (19\%), and macrolides (22\%). From 2002 to 2007, after publication of AAP guide-

FIGURE 2 prescribed in 1998-2007. $P=.71$ for trend. model, time period, gender, race, physician specialty, and age were at least nominally associated $(P<.2)$ with the dependent variable of amoxicillin prescribing. In a model including age and excluding specialty, time period and age were each found to be independently associated with amoxicillin prescribing. On average, each 2-year increase after 1998-1999 was independently associated with a $49 \%$ increase in the odds of amoxicillin prescribing (odds ratio [OR] per 2 years: 1.49 [95\% Cl: 1.16-1.92]). In addition, children 0 to 5 years of age were more likely to receive amoxicillin than were children 12 to 17 years of age (OR: 2.51 [95\% Cl: 1.15-5.47]). When physician specialty was included and patient age excluded, family practitioners were less likely than pediatricians to prescribe amoxicillin (OR: 0.38 [95\% $\mathrm{Cl}$ : $0.16-0.88]$ ).

lines, macrolides constituted 18\% overall antibiotic prescriptions for acute sinusitis.

\section{Factors Associated With Amoxicillin Prescribing}

Among the variables considered for inclusion in the multivariate logistic

\section{DISCUSSION}

In this study, we used a nationally representative data set regarding ambulatory visits to examine time trends in visit rates and antibiotic prescribing

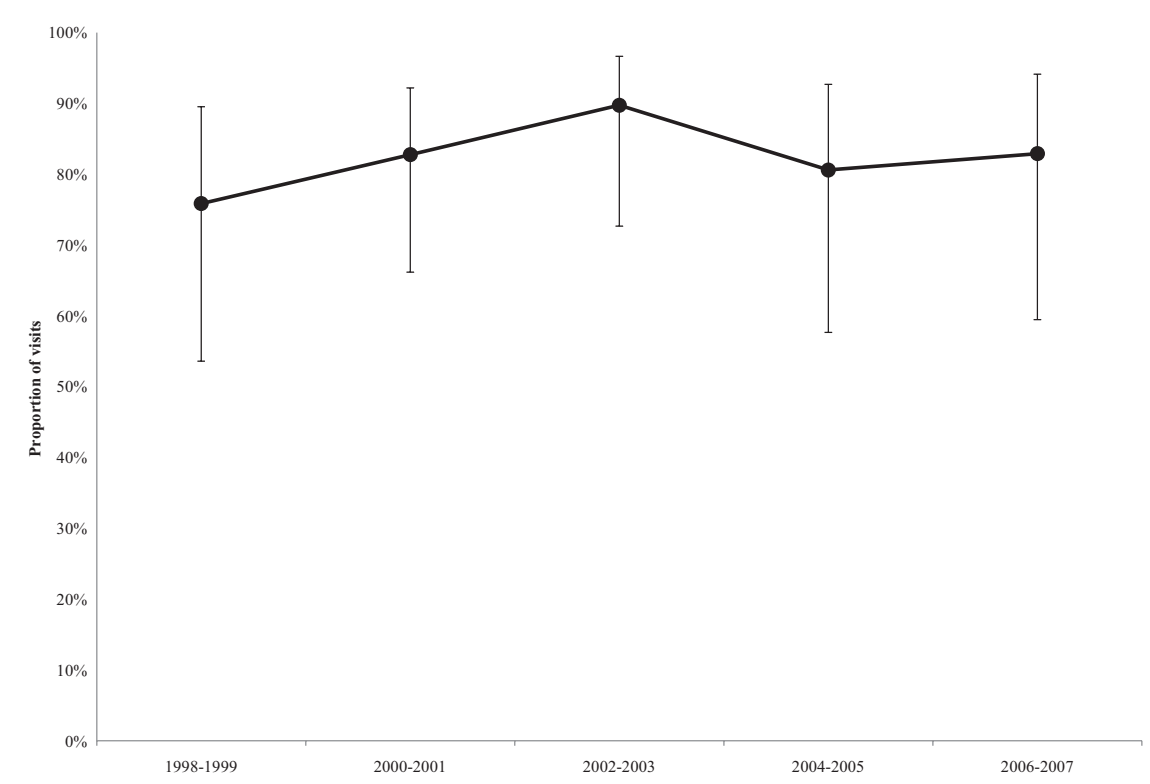

Proportions (estimates and $95 \% \mathrm{Cls}$ ) of visits attributable to acute sinusitis in which antibiotics were 


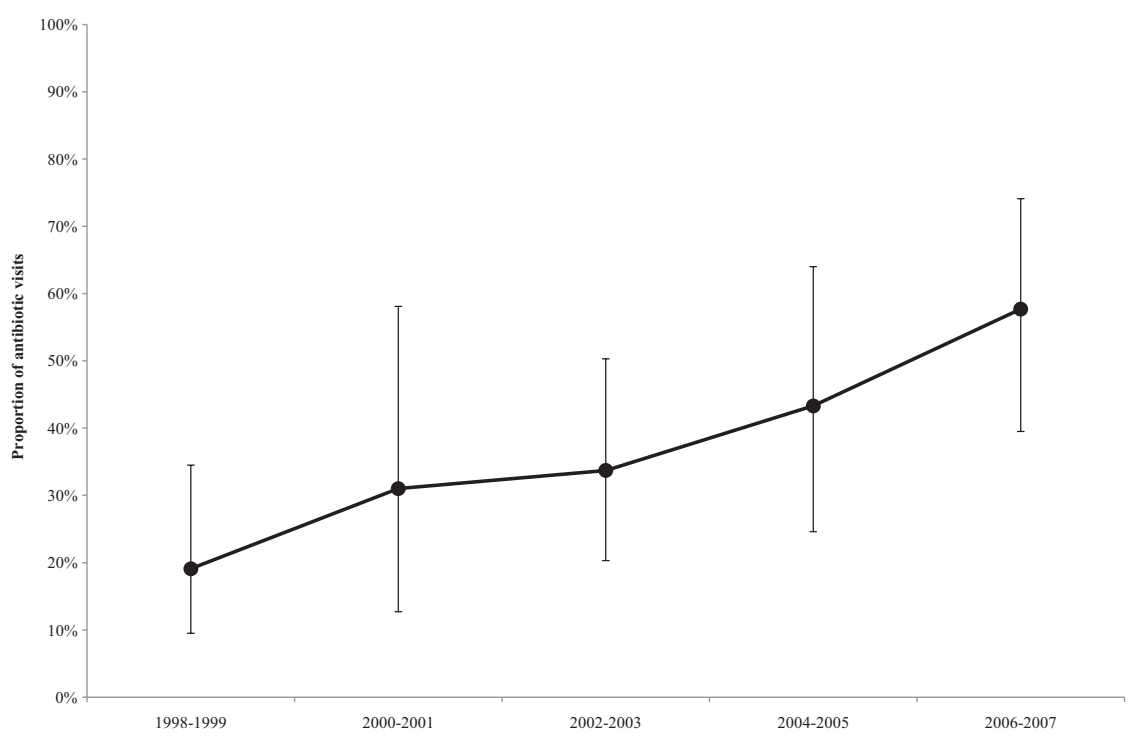

FIGURE 3

Proportions (estimates and $95 \% \mathrm{Cls}$ ) of antibiotic visits attributable to acute sinusitis in which amoxicillin was prescribed in 1998-2007. $P=.01$ for trend.

for children with acute sinusitis. Unlike the observed decrease in the visit rate for A0M during the post-PCV7 period, ${ }^{3}$ the visit rate for acute sinusitis among children did not change between 1998 and 2007. During the same period, and in accordance with the publication of AAP clinical practice guidelines for acute sinusitis in 2001, use of amoxicillin for acute sinusitis increased substantially. This change was similar to the change in prescribing for AOM after the publication of guidelines in $2004 .{ }^{9}$ In addition, we found that pediatric specialty and younger patient age were associated with amoxicillin prescribing for acute sinusitis. Although the increased use of amoxicillin is consistent with recommendations from AAP guidelines, we also found that use of macrolides remained common for acute sinusitis.

Our findings with respect to visit rates for acute sinusitis may be explained by differences in how physicians diagnose acute sinusitis, compared with AOM. Whereas the diagnosis of AOM involves both clinical history findings and distinct physical examination findings, ${ }^{6}$ acute sinusitis is frequently di- agnosed on the basis of the patient's history alone. ${ }^{5,6,15}$ Furthermore, physicians disagree regarding which symptoms define a clinical history suggesting acute sinusitis. In a recent survey of pediatricians, most respondents thought that prolonged symptoms represented an important sign of acute sinusitis, but they varied in their responses regarding the importance of specific symptoms (eg, purulent rhinorrhea) or symptom combinations in establishing a diagnosis. ${ }^{16}$ The absence of a reliable, specific, physical finding that defines acute sinusitis inevitably leads to misclassification of children with viral upper respiratory tract infections as having acute sinusitis. As a result, recent practice guidelines and campaigns for judicious management of respiratory infections (eg, the Centers for Disease Control and Prevention Get Smart Campaign ${ }^{17}$ ) may be more effective in influencing physicians to diagnose AOM more stringently (eg, excluding otitis media with effusion) than is possible for acute sinusitis. Studies using administrative data found that, after introduction of PCV7, the visit rate for AOM de- creased by an amount that exceeded the estimated efficacy of the vaccine, which suggests that changes in how physicians diagnose AOM may account for a substantial proportion of the observed decrease in AOM visits. 7,8

Although there is mixed evidence regarding the effectiveness of antibiotics for treatment of acute sinusitis in children, ${ }^{18-20}$ physicians routinely prescribe antibiotics for treatment of acute sinusitis, and this practice has not changed in the past decade. The proportion of visits in which an antibiotic was prescribed for acute sinusitis in children alone is similar to the rate estimated for children and adults combined in a previous study. ${ }^{1}$ This is also similar to the proportion of visits that result in an antibiotic prescription for $\mathrm{AOM},{ }^{9}$ for which efforts to reduce antibiotic use through observation and delayed prescribing seem to have had limited success. ${ }^{9,21}$ To continue to promote judicious antibiotic prescribing for these infections, novel strategies to enhance the acceptability of such practices likely are needed.

Although prescribing of amoxicillin for acute sinusitis seemed to have begun to increase even before guideline publication in 2001, we found that it continued to increase substantially after the publication of AAP practice guidelines in 2001. A similar association between the timing of guideline publication and changes in antibiotic selection for $\mathrm{AOM}$ was noted in a recent publication that used the same administrative data set as analyzed in this study. ${ }^{9}$ The use of amoxicillin increased in the 2 years after publication of the AAP guidelines for AOM in 2004, reversing an earlier trend of increased use of broadspectrum agents. ${ }^{10}$ Guidelines that are well disseminated, are consistent with physicians' beliefs, ${ }^{22}$ and provide epidemiologically sound rationale can be effective tools in influencing and/or re- 
inforcing prescribing behavior, especially for infectious diseases. ${ }^{23}$

In separate multivariate models that included either patient age or physician specialty among independent variables, we found that younger patient age and pediatric specialty were independently associated with greater odds of amoxicillin use for acute sinusitis. Because colinearity precluded the simultaneous inclusion of these variables in a single model, we were unable to determine fully the extent to which either or both of these factors were associated with amoxicillin use. It is possible that younger children are more likely than older children to receive amoxicillin, independent of specialty, because of differences in the clinical presentation of acute sinusitis between age groups. Younger children may experience symptoms of acute sinusitis that are less specific or less severe than those experienced by older children, in part because of the existence of only partially developed frontal sinuses and a higher incidence of viral infections in this population. ${ }^{24-26}$ For these reasons, physicians may base the diagnosis and corresponding treatment of acute sinusitis in this patient population on different criteria than they would use for older children. ${ }^{16}$ Undeveloped frontal sinuses also make younger children less likely to develop intracranial complications, ${ }^{27}$ which perhaps encourages prescription of a narrow-spectrum agent such as amoxicillin for this group. Conversely, pediatricians might be more likely to prescribe amoxicillin because of a greater influence of AAP guidelines on their practices. Notably, however, a previous study found no difference between pediatricians and family practitioners in their antibioticprescribing patterns for A0M. ${ }^{10}$

Although we examined antibioticselection trends for acute sinusitis in the context of current guidelines recommending amoxicillin as the first-line agent, the appropriate empiric antibiotic agent remains somewhat uncertain. An increase in nontypeable Haemophilus influenzae as a causative organism may make amoxicillinclavulanate a more-appropriate empiric choice than amoxicillin in some communities. ${ }^{4}$ In addition, a recently published clinical trial showed that use of amoxicillin-clavulanate, compared with placebo, was effective in resolving symptoms for stringently diagnosed acute sinusitis in children. ${ }^{20}$ These epidemiological and clinical factors will need to be considered for future updates in clinical guidelines for acute sinusitis.

The frequent use of macrolides for treatment of acute sinusitis, as shown in this study, is potentially problematic because macrolide resistance among $S$ pneumoniae in the United States is common. ${ }^{28}$ Previous studies showed increased use of macrolides for children overall ${ }^{29}$ and for children with A0M. ${ }^{10}$ This study confirms that reducing unnecessary use of macrolides for pediatric upper respiratory tract infections is an important target for improvement in antibiotic prescribing.

We acknowledge limitations to this study. First, our relatively small sample size limited the power of certain statistical analyses. For example, we might not have been able to detect small trends in visit rates for acute sinusitis from this data set, either for children overall or for specific age groups, and our multivariate model may lack the power to detect additional factors associated with amoxicillin prescribing. In time-trend analyses, some periods have fewer than 30 observations, which may yield unstable results. However, when combined into two 5-year periods with larger sample sizes, the observed trends were nearly identical. In addition, variation exists in how physicians diagnose acute sinusitis. ${ }^{16}$ Because we used administrative data, we were not able to verify specific symptoms associated with our sampled visits and thus were unable to measure the degree to which these characteristics of our sample correlate with those outlined in published national guidelines.

\section{CONCLUSIONS}

Despite these limitations, our findings have several implications. Although acute sinusitis and AOM often are considered similar diseases, ${ }^{30}$ this study highlights trends in ambulatory visit rates and antibiotic treatment patterns for acute sinusitis that are different from those observed for AOM during the post-PCV7 era. Because of the continued tendency of physicians to prescribe antibiotics for treatment of acute sinusitis, this condition remains an important target for campaigns promoting judicious antibiotic use. Our findings also provide support for continued efforts to develop and to disseminate clinical practice guidelines as a tool to optimize antibioticprescribing practices.

\section{ACKNOWLEDGMENTS}

Dr Hersh was supported by National Institutes of Health grants T32HD044331 and KL2 RR02413.

Amy Markowitz provided helpful comments in drafting the final manuscript.

\section{REFERENCES}

1. Sharp HJ, Denman D, Puumala S, Leopold DA. Treatment of acute and chronic rhinosinusitis in the United States, 1999-2002.

Arch Otolaryngol Head Neck Surg. 2007; 133(3):260-265

2. McCaig LF, Besser RE, Hughes JM. Trends in an- timicrobial prescribing rates for children and adolescents. JAMA. 2002;287(23):3096-3102

3. Grijalva CG, Nuorti JP, Griffin MR. Antibiotic 
prescription rates for acute respiratory tract infections in US ambulatory settings. JAMA. 2009;302(7):758-766

4. Brook I. Current issues in the management of acute bacterial sinusitis in children. Int $J$ Pediatr Otorhinolaryngol. 2007;71(11): 1653-1661

5. American Academy of Pediatrics, Subcommittee on Management of Sinusitis and Committee on Quality Improvement. Clinical practice guideline: management of sinusitis [published corrections appear in Pediatrics. 2002;109(5):40 and Pediatrics. 2001; 108(5):A24]. Pediatrics. 2001;108(3): 798-808

6. American Academy of Pediatrics, Subcommittee on Management of Acute Otitis Media. Diagnosis and management of acute otitis media. Pediatrics. 2004;113(5): 1451-1465

7. Zhou F, Shefer A, Kong Y, Nuorti JP. Trends in acute otitis media-related health care utilization by privately insured young children in the United States, 1997-2004. Pediatrics. 2008;121 (2):253-260

8. Grijalva CG, Poehling KA, Nuorti JP, et al. National impact of universal childhood immunization with pneumococcal conjugate vaccine on outpatient medical care visits in the United States. Pediatrics. 2006;118(3): 865-873

9. Coco A, Vernacchio L, Horst M, Anderson A. Management of acute otitis media after publication of the 2004 AAP and AAFP clinical practice guideline. Pediatrics. 2010; 125(2): $214-220$

10. Coco AS, Horst MA, Gambler AS. Trends in broad-spectrum antibiotic prescribing for children with acute otitis media in the United States, 1998-2004. BMC Pediatr. 2009;9:41

11. National Center for Health Statistics. International Classification of Diseases, Ninth Revision, Clinical Modification (ICD-9-CM).
Available at: www.cdc.gov/nchs/icd/ icd9cm.htm. Accessed March 30, 2009

12. National Center for Health Statistics. About the Ambulatory Health Care Surveys. Available at: www.cdc.gov/nchs/ahcd/about_ ahcd.htm. Accessed March 30, 2010

13. National Center for Health Statistics. Ambulatory Care Drug Database System. Available at: www.cdc.gov/nchs/ahcd/ahcd_ database.htm. Accessed March 30, 2010

14. US Census Bureau. Population estimates: national characteristics. Available at: www. census.gov/popest/national/asrh. Accessed February 2, 2010

15. Dowell SF, Schwartz B, Phillips WR. Appropriate use of antibiotics for URIs in children, part I: otitis media and acute sinusitis. Am Fam Physician. 1998;58(5):1113-1118, 1123

16. McQuillan L, Crane LA, Kempe A. Diagnosis and management of acute sinusitis by pediatricians. Pediatrics. 2009;123(2). Available at: www.pediatrics.org/cogi/content/full/123/ 2/e193

17. Centers for Disease Control and Prevention. Get Smart: know when antibiotics work. Available at: www.cdc.gov/getsmart/index. html. Accessed March 30, 2010

18. Wald ER, Chiponis D, Ledesma-Medina J. Comparative effectiveness of amoxicillin and amoxicillin-clavulanate potassium in acute paranasal sinus infections in children: a double-blind, placebo-controlled trial. Pediatrics. 1986;77 (6):795-800

19. Garbutt JM, Goldstein M, Gellman E, Shannon W, Littenberg B. A randomized, placebocontrolled trial of antimicrobial treatment for children with clinically diagnosed acute sinusitis. Pediatrics. 2001;107 (4):619-625

20. Wald ER, Nash D, Eickhoff J. Effectiveness of amoxicillin/clavulanate potassium in the treatment of acute bacterial sinusitis in children. Pediatrics. 2009;124(1):9-15

21. Vernacchio L, Vezina RM, Mitchell AA. Man- agement of acute otitis media by primary care physicians: trends since the release of the 2004 American Academy of Pediatrics/ American Academy of Family Physicians clinical practice guideline. Pediatrics. 2007; 120(2):281-287

22. Cabana MD, Rand CS, Powe NR, et al. Why don't physicians follow clinical practice guidelines? A framework for improvement. JAMA. 1999;282(15):1458-1465

23. Hersh AL, Maselli JH, Cabana MD. Changes in prescribing of antiviral medications for influenza associated with new treatment guidelines. Am J Public Health. 2009; 99(suppl 2):S362-S364

24. Incaudo GA, Wooding LG. Diagnosis and treatment of acute and subacute sinusitis in children and adults. Clin Rev Allergy Immunol. 1998;16(1-2):157-204

25. Kogutt MS, Swischuk LE. Diagnosis of sinusitis in infants and children. Pediatrics. 1973; 52(1):121-124

26. Wald ER, Guerra N, Byers C. Upper respiratory tract infections in young children: duration of and frequency of complications. Pediatrics. 1991;87(2):129-133

27. Herrmann BW, Chung JC, Eisenbeis JF, Forsen JW Jr. Intracranial complications of pediatric frontal rhinosinusitis. Am J Rhinol. 2006;20(3):320-324

28. Doern GV, Richter SS, Miller A, et al. Antimicrobial resistance among Streptococcus pneumoniae in the United States: have we begun to turn the corner on resistance to certain antimicrobial classes? Clin Infect Dis. 2005;41(2): 139-148

29. Stille CJ, Andrade SE, Huang SS, et al. Increased use of second-generation macrolide antibiotics for children in nine health plans in the United States. Pediatrics. 2004; 114(5):1206-1211

30. Parsons DS, Wald ER. Otitis media and sinusitis: similar diseases. Otolaryngol Clin North Am. 1996;29(1):11-25 